\title{
THE ESTIMATION OF IRON BY PERMANGANATE IN PRESENCE OF HYDROCHLORIC ACID.
}

\author{
By G. CECIL JONES, A.C.G.I., F.I.C., AND JOHN H. JEFFERY. \\ (Read at the Meeting, June 9, 1909.)
}

IN most textbooks of analytical chemistry, two methods are described for the estimation of iron by permanganate in presence of hydrochloric acid-namely, the method of Fresenius and that of Zimmermann-Reinhardt. Within the last year or so doubt has been cast on both these methods, Harrison and Perkin (Analyst, 1908, 33, 47), as a result of their experiments with the Zimmermann-Reinhardt method, expressing the view that "for exact titrations permanganate cannot be employed in presence of hydrochloric acid," whilst Birch (Chem. News, 1909, 99, 61 and 73) was unable to confirm the results of Fresenius.

Had we felt that Harrison and Perkin were justified in their conclusion, this paper would never have been written; but their work certainly showed that very inaccurate results might be obtained by following Treadwell's description of Reinhardt's method, and suggested that the excellent results published by earlier authors might have been due to a happy balance of opposing errors. On the other hand, it remained possible that the other workers had, in their experiments, secured constancy of certain conditions, the importance of which they did not realise, or at least failed to emphasise. We set ourselves the task of determining whether any set of conditions, reasonably attainable, would render the Zimmermann-Reinhardt method of universal application, whilst we were led to study Fresenius's method by the recommendation given to it by Hehner during the discussion of Harrison and Perkin's paper.

It will be convenient to review briefly, in the light of our present knowledge, the history of these methods, more especially as that of Fresenius, though supported for a generation by his great name, finds little or no support in the experiments which he describes.

When Marguerite (Ann. Chim. Phys., 1846, 18, 244) first recommended the use of permanganate for the volumetric determination of iron, it was supposed that the reaction proceeded quantitatively in accordance with the equation

$$
\text { (1) } 10 \mathrm{FeO}+\mathrm{Mn}_{2} \mathrm{O}_{7}=5 \mathrm{Fe}_{2} \mathrm{O}_{3}+2 \mathrm{MnO} \text {, }
$$

whether the titration was conducted in presence of sulphuric acid or of hydrochloric acid. Löwenthal and Lenssen (Zeit. anal. Chem., 1862, 1, 329) were the first to show that, in the presence of hydrochloric acid, the consumption of permanganate was excessive, and that this was due to its being used up in oxidising hydrochloric acid in accordance with the equation

$$
\text { (2) } 10 \mathrm{HCl}+\mathrm{Mn}_{2} \mathrm{O}_{7}=5 \mathrm{Cl}_{2}+2 \mathrm{MnO}+5 \mathrm{H}_{2} \mathrm{O} \text {, }
$$

a reaction which proceeds but slowly in pure dilute solutions, but which is greatly accelerated by the presence of iron salts. There is some evidence to-day that the 
reaction may be less simple than is indicated by equation (2), but the net result of the possible series of reactions is reduction of permanganate and liberation of chlorine, some of which escapes reduction by the ferrous iron, the exact amount of such escape depending on the conditions of the experiment. From a study of the results of Löwenthal and Lenssen, which he repeated and confirmed, Fresenius (Zeit. anal. Chem., 1862, 1, 361) was led to recommend the method known by his name. His experiments were of this kind : He found that 25 c.c. of $\frac{\mathrm{N}}{10}$ ferrous solution, diluted to a litre with water containing some 30 c.c. of hydrochloric acid (specific gravity $1 \cdot 1)$, required the addition of something like 26 c.c. of $\frac{\mathrm{N}}{10}$ permanganate before the pink tint of the latter became permanent. If then a further 25 c.c. of ferrous solution was added to the contents of the flask or basin and the titration continued, not much more than 25 c.c. of permanganate was usually necessary to restore the pink colour, whilst a third addition of ferrous solution almost invariably required exactly 25 c.c. of permanganate. On the strength of these experiments, Fresenius recommended that, where titration in presence of hydrochloric acid was unavoidable, the iron solution should be made up to 250 c.c., 50 c.c. of this diluted with a large volume of water, and permanganate added until a permanent pink resulted, when a further 50 c.c. of the iron solution was to be added and titration continued, and so on. When two consecutive and closely concordant numbers were obtained, the mean of these was to be taken as the true reading for 50 c.c. of the iron solution. According to Fresenius, the third and fourth numbers should always, and the second and third usually, agree well together, but he gives no practical example of the application of this, his first, method. A second method, where but a small quantity of the ferrous solution was available, was to add to a considerable volume of water, acidified with hydrochloric acid, some 25 c.c. of $\frac{N}{10}$ ferrous solution and just sufficient permanganate to produce a permanent pink tint, and then to pour into this mixture the liquid to be analysed, when titration with permanganate was said to give a fair result. Two examples of this method were given, but we show later that an important condition was realised in these experiments which would never be the case in any practical attempt to apply the method.

Kessler (Pogg. Ann., 1863, 119, 225) recorded the fact that in the presence of much sulphuric acid, and still more in that of manganese sulphate, the disturbing influence of hydrochloric acid is greatly reduced; but it remained for Zimmermann (Annalen, 1882, 213, 305; also Ber. deut. chem. Ges., 1881, 14, 779) to recommend the use of manganese sulphate as an aid to accuracy of analysis. $\mathrm{He}$ stated that, if at least 1 gram of the crystallised salt were taken for each 15 c.c. of hydrochloric acid (specific gravity 1.1) supposed to be present, iron might be titrated as accurately in hydrochloric as in sulphuric acid solution. Reinhardt (Chem. Zeit., $1889,13,323)$ replaced the simple manganese sulphate solution of Zimmermann by a solution made by dissolving 200 grams of the crystallised salt in 1,000 c.c. of water, and adding to this a cooled mixture of 400 c.c. concentrated sulphuric acid, 600 c.c. water, and 1,000 c.c. phosphoric acid (specific gravity 1-3). The use of this solution, of which Reinhardt took 60 c.c. for each experiment, renders the assay liquid practically colourless until the permanent pink tint due to permanganate announces the completion of the titration. In the absence of phosphoric acid, the 
yellow colour of ferric chloride renders the end-point less clear. The addition of phosphoric acid to the manganese sulphate appears also to increase slightly the efficiency of the latter as a retarder of chlorine evolution. Reinhardt says nothing concerning the accuracy of the method.

Since the introduction of Reinhardt's modification of Zimmermann's method, it has been usual to speak of titrations conducted in absence of hydrochloric acid, and in which Marguerite's equation (1) is strictly fulfilled, as being carried out according to Marguerite, and the use of the expression "Marguerite's method" in this restricted sense will save much verbal repetition in the paragraphs which follow.

Treadwell ("Analytical Chemistry," vol. ii., English edition, 483), who makes use of Reinhardt's solution, states that the amount of manganous salt present should not exceed the amount of iron to be estimated. We have been unable to discover any publication of his in which he gives grounds for this statement, but possibly it was the result of an experience similar to that of Harrison and Perkin. These authors (loc. cit.) found that Reinhardt's solution itself may exert an oxidising action on ferrous solutions, and so reduce the permanganate consumption; and that this oxidising power is not even constant, but is in some way dependent on the age of the solution. The results they obtained by following Treadwell's directions varied considerably, but were all too high, and the best of them were found to owe their relative accuracy to the oxidising power of the newly prepared Reinhardt solution. Working with older manganese solutions, they found the over-consumption of permanganate as much as 0.8 c.c. with only 15 c.c. of hydrochloric acid (specific gravity $1 \cdot 1$ ) present, and the over-consumption increased with increasing concentration of hydrochloric acid.

On the other hand, Brandt (Chem. Zeit., 1908, 32, 812, 830, 840, and 851) gives tables showing that in his hands the method of Reinhardt yields results which, if not wholly independent of the amount of hydrochloric acid present, are at any rate not greatly influenced by much larger quantities than those employed by Harrison and Perkin. The main purpose of his paper is the recommendation of pure ferric oxide as a standard for determining the titre of permanganate solutions intended for use in estimating iron by the Zimmermann-Reinhardt method, and, if this substance be adopted as the standard, dissolved in hydrochloric acid and reduced by stannous chloride precisely as an ore would be treated, he says that method leaves nothing to be desired. His experiments show that, under the conditions which prevail in his practice, and with iron equivalent to about 50 c.c. of a decinormal solution present, the permanganate consumption is almost exactly $0^{\circ} 2$ c.c. greater than is required by Marguerite's equation. For routine analysis, where approximately the same reading-40 to 50 c.c.-can be obtained in every experiment, no correction is necessary if ferric oxide be adopted as the standard. His paper provides no answer to the criticisms of Harrison and Perkin concerning the alterability of Reinhardt's solution, and it is strange that he did not think to vary the amount of iron in his experiments, which give one no hint as to the interpretation to be placed on a reading of a different order of magnitude, say, 2.5 c.c. If 0.498 gram of pure ferric oxide, reduced and titrated by Reinhardt's method, gives a reading of 50 c.c., to how much ferric oxide does a reading of 2.5 c.c. correspond? One-twentieth of 0.498 is 
0.0249 , or, say, 0.025 ; but Brandt tells us that the error of the method, as compared with that of Marguerite, is $0 \cdot 2$ c.c. when there is ferrous iron present equivalent to 0.498 gram of ferric oxide, and the error will surely not be less when the concentration of iron is reduced, and that of hydrochloric acid maintained constant. It would seem more reasonable to suppose that 2.5 c.c. was equivalent to 0.023 gram ferric oxide, and without experiment no one would care to assume that it had a value even so high as this. Now, the difference between 0.025 and 0.023 is a matter of 10 per cent., far too large an amount to be left in doubt.

To the iron and steel worker these considerations will have no weight. He can insure a reading of 40 to 50 c.c., and, moreover, is too expert in the use of bichromate to heed our results or Brandt's; if, indeed, he has not as a rule a method of his own for applying permanganate. With the general worker it is far otherwise. He is called upon to estimate widely varying quantities of iron in materials, as to the composition of which he knows very little in advance; and there is no doubt that he would as a rule prefer to use permanganate were he sure that it would give him accurate results, and it is to such as he that this paper is addressed.

\section{EXPERIMENTAL.}

We have reduced our experimental record to just so much as is necessary to support our conclusions and to enable anyone to repeat our more instructive experiments. The following tables are compiled from experiments made within the last few days with the special object of saving our readers' time. The results published here find confirmation in many other series of experiments, made with solutions not exactly equivalent, and therefore requiring closer inspection to learn their bearing than the busy reader can well spare. Needless to say, many of our early series of experiments, made when our knowledge was less complete, are less regular; but our purpose is to show how permanganate can be used in presence of hydrochloric acid, and not how we obtained this knowledge.

The following solutions were used :

Manganese Solution: Reinhardt's solution, the preparation of which has already been described.

Stannous Chloride: 50 grams of the crystallised salt and 100 c.c. of concentrated hydrochloric acid made up to 1,000 c.c.

Mercuric Chloride: A cold saturated solution.

Hydrochloric Acid: 6-normal, that is to say, acid of specific gravity $1 \cdot 1$, or about the strength of the mixture which exhibits a constant boiling-point.

Sulphuric Acid: Also, for convenience of comparison, about 6-normal.

In each of the experiments recorded in Table I., the quantity of water stated in column B was mixed in a capacious basin with the Reinhardt solution, where this was used, and tinted with permanganate, of which 1 drop sufficed in every case; 25 c.c. of a freshly prepared solution of ferrous ammonium sulphate was then added, and simultaneously the acid, hydrochloric or sulphuric, as the case might be. Where stannous chloride or mercuric chloride were used, these were added with the acid as follows: Ten minutes before each titration was made, the requisite amount of 
hydrochloric acid was placed in a flask, and the stannous chloride and mercuric chloride added. This mixture was added to the contents of the titration bowl simultaneously with the ferrous solution. The permanganate was then allowed to fall drop by drop into the bowl with constant stirring, each titration occupying about four minutes. All the numbers in the table in columns A to $\mathrm{H}$ represent cubic centimetres.

TABLE I.

\begin{tabular}{|c|c|c|c|c|c|c|c|c|}
\hline $\begin{array}{l}\text { No. of } \\
\text { Experi- } \\
\text { ment. }\end{array}$ & $\begin{array}{c}\text { A. } \\
\text { Reinhardt's } \\
\text { Solution. }\end{array}$ & $\begin{array}{c}\text { B. } \\
\text { Water. }\end{array}$ & $\begin{array}{c}\text { C. } \\
6 \mathrm{~N} \cdot \mathrm{H}_{2} \mathrm{SO}_{4} .\end{array}$ & $\begin{array}{c}\text { D. } \\
\text { 6N.HCl. }\end{array}$ & $\begin{array}{c}\text { E. } \\
\mathrm{SnCl}_{2} \\
\text { Solution. }\end{array}$ & $\begin{array}{c}\text { F. } \\
\mathrm{HgCl}_{2} \\
\text { Solution. }\end{array}$ & $\begin{array}{c}\text { G. } \\
\text { Final } \\
\text { Volume. }\end{array}$ & $\begin{array}{l}\text { H. } \\
\text { Perman- } \\
\text { ganate } \\
\text { Consump- } \\
\text { tion. }\end{array}$ \\
\hline 1. & - & 425 & 25 & - & - & - & 500 & $25 \cdot 05$ \\
\hline 2. & 25 & 400 & 25 & - & - & - & 500 & $25 \cdot 0$ \\
\hline 3. & 25 & 425 & - & - & - & - & 500 & $25 \cdot 0$ \\
\hline 4. & 50 & 400 & - & - & - & - & 500 & $25 \cdot 0$ \\
\hline 5. & - & 400 & 50 & -- & - & - & 500 & $25 \cdot 0$ \\
\hline 6. & - & 150 & 300 & - & - & - & 500 & $25 \cdot 0$ \\
\hline 7. & - & 425 & - & 25 & $\ldots$ & - & 500 & $25 \cdot 5$ \\
\hline 8. & - & 415 & $\ldots$ & 25 & 0.5 & 10 & 500 & $25 \cdot 7$ \\
\hline 9. & 25 & 400 & - & 25 & - & - & 500 & $25 \cdot 05$ \\
\hline 10. & 25 & 390 & $\ldots$ & 25 & 0.5 & 10 & 500 & $25 \cdot 1$ \\
\hline 11. & 50 & 365 & - & 25 & 0.5 & 10 & 500 & $25 \cdot 1$ \\
\hline 12. & 10 & 405 & - & 25 & 0.5 & 10 & 500 & $25 \cdot 2$ \\
\hline 13. & 25 & 340 & - & 25 & 0.5 & 60 & 500 & $25 \cdot 1$ \\
\hline 14. & 25 & 390 & - & 25 & - & 10 & 500 & 25.05 \\
\hline 15. & 25 & 365 & - & 50 & 0.5 & 10 & 500 & $25 \cdot 15$ \\
\hline 16. & 50 & 340 & - & 50 & 0.5 & 10 & 500 & $25 \cdot 1$ \\
\hline 17. & 25 & 405 & - & 10 & 0.5 & 10 & 500 & $25 \cdot 05$ \\
\hline 18. & 25 & 390 & - & 25 & $1 \cdot 0$ & 10 & 500 & $25 \cdot 15$ \\
\hline 19. & 25 & 390 & - & 25 & $2 \cdot 0$ & 10 & 500 & $25 \cdot 25$ \\
\hline 20. & 25 & 900 & - & 25 & 0.5 & 10 & 1000 & $25 \cdot 1$ \\
\hline 21. & 25 & 1400 & - & 25 & 0.5 & 10 & 1500 & $25 \cdot 15$ \\
\hline 22. & 25 & 90 & - & 25 & 0.5 & 10 & 200 & 25.05 \\
\hline
\end{tabular}

Experiments 2 and 3 compared with 1 appear to confirm the observation of Harrison and Perkin that Reinhardt's solution exerts an oxidising action on the iron solution; but the solution used in this particular series of experiments had no such action as experiment 4 shows, where doubling the volume of Reinhardt's solution does not affect the result. Had the sulphuric acid contained ferrous iron, as it frequently does, experiments 5 and 6 would have shown higher results than experiment 1. That they give identical numbers, higher than that found in experiment 1 , only illustrates the fact to which Treadwell has already drawn attention, that to obtain accurate results by Marguerite's method, it is necessary to have much sulphuric acid present, the minimum allowable strength being about half-normal. Larger quantities of sulphuric acid are without influence on the result. 
Experiment 7 may appear a surprisingly good result to some who have studied the influence of hydrochloric acid on permanganate titrations of iron. Harrison and Perkin record an excess consumption of permanganate of 0.6 c.c., with only onefourth as much hydrochloric acid present. The difference between their results and ours is, we believe, due to difference in manner of adäing the permanganate. By running this in in a continuous stream up to 23 c.c. and then proceeding by drops, we have obtained results practically identical with theirs. Experiment 8 shows that either stannic or mercuric or mercurous chloride, all of which are present in practical titrations, reinforce the disturbing action of the hydrochloric acid. Experiment 9 shows that 25 c.c. of Reinhardt's solution reduces the error introduced by 25 c.c. of hydrochloric acid to 0.05 c.c., whilst experiment 10 shows that it does not wholly neutralise the action of the tin or mercury salts. Experiments 11 and 12 show that, while no advantage is gained by increasing the proportion of Reinhardt's solution, it is not wise to diminish it greatly below that used in experiment 10 . Experiment 13 shows that no advantage attends the use of large quantities of mercuric chloride, such as the 60 c.c. used by Reinhardt and by most workers since his time. Experiments 15 and 16 show that doubling the amount of hydrochloric acid, whilst. preserving unaltered all the other conditions, increases the over-consumption of permanganate from 0.1 to 0.15 , but that this can be prevented from exceeding 0.1 by taking more of Reinhardt's solution. Experiment 17 shows how the overconsumption of permanganate falls when the amount of hydrochloric acid is greatly reduced. Experiment 14, compared with No. 9, shows that mercuric chloride, as might be expected, has no influence on the titration, whilst Nos. 18 and 19 show that either stannic or mercurous chloride has a notable influence. Experiment 20 shows that dilution considerably beyond 500 c.c. is without influence, whilst Nos. 21 and 22, like the figures of Zimmermann, Harrison and Perkin, and Birch, directly challenge the recommendation to be found in all the textbooks from the time of Fresenius that the titration is best conducted in highly dilute solution. The greater the dilution, the wider the divergence from Marguerite's equation. In practice, however, a final volume of about 500 c.c. is preferable, since comparatively wide variations from this volume have no measurable influence on the result. In a bulk of 1,500 c.c., such as Reinhardt recommended, one drop of permanganate is scarcely enough to produce a distinct pink tint, whilst in small bulks, under 200 c.c., though with great care the absolute error may be very slightly reduced, it is more difficult to get closely concordant duplicates, especially if the amount of hydrochloric acid is large.

A number of the experiments of Table I. were next repeated with the following modification: The measured 25 c.c. of ferrous solution was completely oxidised, mixed with the quantity of hydrochloric acid stated in column D, heated to boiling, and reduced by stannous chloride, which towards the end was added 2 drops at a time. When 2 drops of stannous chloride produced no further change of tint, a measured excess of the reagent was added, the solution cooled, and subsequently mixed with mercuric chloride. After ten minutes, the contents of the flask were emptied into a bowl containing the Reinhardt solution and water previously tinted by 1 drop of permanganate. The results of experiments 10 to 13 and 15 to 19 were 
confirmed in every case. Since the experiments of this series differed from those in Table I. by the presence of some 25 c.c. of $\frac{N}{10}$ stannic chloride, it may be taken that this reagent has, as might be expected, no influence on the course of the titration. On the contrary, it seems to be conclusively proved that mercurous chloride has a marked influence if present in large amount. The excess of stannous chloride can easily be kept below 0.25 c.c., and the results differ inappreciably when the excess is varied from $0 \cdot 1$ to 0.5 c.c. The interval of ten minutes between the addition of the mercuric chloride and the commencement of the titration is the result of our observation that, when the excess of stannous chloride is purposely kept very small, the precipitate of mercuric chloride takes an appreciable time to appear. From this we were led to suppose that the reaction $\mathrm{SnCl}_{2}+2 \mathrm{HgCl}_{2}=\mathrm{SnCl}_{4}+2 \mathrm{HgCl}$ might require an appreciable time to approximate completion, and this we find to be the case. Titration of the liquid immediately after the addition of the mercuric chloride leads to high results, due to the presence of unoxidised stannous chloride. We find that Reinhardt knew that the reaction $\mathrm{SnCl}_{2}+2 \mathrm{HgCl}_{2}=\mathrm{SnCl}_{4}+2 \mathrm{HgCl}$ never even approximated completion in dilute solutions, and he insisted on the addition of the mercuric chloride before diluting largely, but even in a bulk of 100 c.c. the reaction is slow. Probably ten minutes is an unnecessary allowance, but it has been found to be both ample and safe, since no appreciable oxidation of iron takes place in a much longer time, identical results being obtained after thirty minutes' standing.

In a further series of experiments, recorded in Table II., varying quantities of ferrous solution were titrated with permanganate under the conditions indicated in experiment 10 of Table I. Here, again, two methods were followed-namely, (1) Simple mixture of the ferrous solution with hydrochloric acid, mercurous and mercuric chloride, followed by titration in presence of Reinhardt's solution; and (2) titration of the ferrous solution by permanganate in presence of a little sulphuric acid, reduction of the resulting ferric solution by stannous chloride in presence of 25 c.c. of hydrochloric acid (specific gravity $1 \cdot 1$ ), treatment with mercuric chloride, and re-titration by Reinhardt's method. Had we contented ourselves with one of these methods only, our conclusions might perhaps have dissatisfied some; but, fortunately, the two methods give identical results. Table II. shows the permanganate consumption $(a)$ in half-normal sulphuric acid solution, and $(b)$ by Reinhardt's method.

\section{TABLE II.}

$\begin{array}{ccc}\frac{\mathrm{N}}{10} \text { Ferrous Solution. } & \text { (a) }{\mathrm{In} \mathrm{H}_{2} \mathrm{SO}_{4}}_{50}^{\text {Consumption of }} & \overbrace{\text { Solution. }}^{\frac{\mathrm{N}}{10} \text { Permanganate. }} \\ 20 & 50 & 50 \cdot 1 \\ 10 & 20 & 20 \cdot 1 \\ 5 & 10 & 10 \cdot 1 \\ 2 & 5 & 5 \cdot 1 \\ 1 & 2 & 2 \cdot 1 \\ & 1 & 1 \cdot 1\end{array}$

We think these results show plainly that the small unavoidable error introduced by hydrochloric acid, even in presence of Reinhardt's solution, is a constant one, 
independent of the amount of iron present, and therefore easily corrected for. The correction, though small, cannot be omitted, as on a small amount of iron it represents a notable percentage.

We have made many preparations of Reinhardt's solution, and, though we have occasionally found them to possess oxidising power when first made, this has never been so great as that observed by Harrison and Perkin. Moreover, before the solutions are a week old this oxidising power becomes so small as to be unmeasurable. It is true that we used the purest chemicals procurable, but since we have found it possible to cut down the quantities to be used in each experiment to less than half those proposed by Reinhardt, the extra expense involved by this is not great.

It is impossible to lay too much stress on the importance of adding the permanganate slowly, drop by drop. We were led to this discovery by differences between ourselves, which by a process of elimination were ultimately traced to the difference in the speed at which we ran in the permanganate. We have since found that this point has been dealt with very fully by Skrabal (Zeit. anal. Chem., 1903, 42, 359). We think the results of Birch and of Harrison and Perkin are evidence that they, too, were ignorant of the work of Skrabal, and this is perhaps not surprising, since the literature of the subject these authors were studying is immense, and in the course of his fifty-page paper Skrabal records but one experiment with Reinhardt's solution, and makes no reference at all to the method of Fresenius. Some of the earliest authors-Löwenthal and Lenssen, for instance-speak of the addition of permanganate "drop by drop slowly" as the custom in their time, but there is no evidence whatever that they realised that such procedure was of vital importance. On the contrary, their own poor results suggest that they themselves disregarded the custom.

For the estimation of iron in ferric oxide, or other substance most easily got into solution in hydrochloric acid, we recommend solution in 25 c.c. of hydrochloric acid (specific gravity $1 \cdot 1$ ), reduction by stannous chloride, using as small an excess of this as is reasonably possible, and addition of 10 c.c. of mercuric chloride solution when cold. Into a capacious bowl we bring 25 c.c. of Reinhardt's solution and some 400 to 500 c.c. of tap-water, and tint the mixture by the addition of permanganate, of which 1 drop should suffice. Ten minutes after the addition of the mercuric chloride to the ferrous solution, we pour the latter into the bowl, rinse the flask or beaker, and add the rinsings to the contents of the bowl, which are then titrated with permanganate, drop by drop, with constant stirring. From the burette reading we deduct 0.1 c.c., and calculate the amount of iron present by reference to the known titre of the permanganate as determined by titration against a ferrous solution free from hydrochloric acid. With the above-mentioned quantities we believe the method capable of giving results equal in accuracy to those obtained by any volumetric method, and it is not difficult to keep all the quantities sufficiently near those named. A final dilution of from 400 to 1,000 c.c. is without measurable influence on the result, and tap-water-that supplied to our laboratory, at any rate -may replace distilled water without disadvantage. This has saved us the trouble of boiling and cooling out of contact with air the large volumes of water required by 
our experiments. The amount of acid need never be much less than 25 c.c., for after solution of the iron oxide it can always be made up to its original volume with acid of constant boiling-point. If, on the other hand, it is necessary to use fuming acid, this will be down to the strength of constant boiling-point acid by the time solution is complete; and if its volume much exceeds 25 c.c., all that is necessary is to add an equal volume of Reinhardt's solution instead of the usual 25 c.c. The tin solution recommended by Treadwell is too strong, having regard to the great influence of relatively small amounts of mercurous chloride. Of our tin solution, it is immaterial whether the excess used is 1 drop or 10-a sufficiently wide margin for anyone. Provided the amount of hydrochloric acid is not less than about 20 c.c. (specific gravity 1.1), and that the volume of Reinhardt solution is at least equal to that of hydrochloric acid, the consumption of permanganate will in every experiment be almost exactly $0 \cdot 1$ c.c. in excess of that demanded by Marguerite's equation. In our hands it averages a little less than this, but is much nearer 0.1 than 0.05 , and we do not feel justified in splitting drops.

There is much to be said for Brandt's proposal to adopt pure ferric oxide as a standard for determining the titre of permanganate solutions; but if this is done, it is preferable to record the true and not the apparent titre on the label, if the solution is to be used for general purposes and not merely for the analysis of ores. For example, if 0.3194 gram pure ferric oxide, by Reinhardt's method, requires exactly 40.0 c.c. of our permanganate, we do not label the solution "exactly decinormal," as Brandt would do, but calculate the true titre as 1 c.c. $=\frac{0.3194}{39.9}$ gram ferric oxide, or
0.2234 $\frac{0.2234}{39.9}$ gram iron. Provided that 0.1 c.c. be always deducted from the observed permanganate consumption, the solution can then be used for the estimation of widely varying quantities of iron by Reinhardt's method.

If the use of freshly made manganese solution is unavoidable, the oxidising power of this can be determined in ten minutes by two experiments, like 3 and 5 in Table I., and allowed for if a measurable quantity.

Unlike Birch, we found no difficulty in repeating the experiments of Fresenius, and even obtained better results than Fresenius himself, due no doubt to the slow speed at which we added the permanganate. When, however, we came to apply Fresenius's recommendations practically, we were scarcely surprised to find the method break down. For in his experiments, after the first titration, Fresenius contented himself with adding more ferrous solution, and made no further addition of acid. Now, the analyst, with his ferrous solution in one flask and hydrochloric acid in the other, would presumably never mix them, and in any practical application of Fresenius's method each addition of ferrous solution would be accompanied by the addition of more acid, and, as a rule, of mercurous chloride, stannous chloride being infinitely more convenient to use than zinc.

The following experiments represent an attempt to apply Fresenius's method under test conditions: 125 c.c. of $\frac{\mathrm{N}}{\mathrm{i} 0}$ ferric chloride, containing hydrochloric acid equivalent to 75 c.c. of constant boiling-point acid, was reduced by stannous chloride, and made up to 250 c.c. with mercuric chloride solution. Successive portions of 50 c.c. were then added to 1,000 c.c. of water and titrated slowly with permanganate. 


\begin{tabular}{|c|c|c|c|c|c|}
\hline 1st 50 & c.c. & juire & $25 \cdot 7$ & c.c. pe & janate \\
\hline 2nd & " &, & $25 \cdot 7$ & ", & , \\
\hline 3rd & ", & ", & $25 \cdot 75$ & , & ", \\
\hline 4 th & ", & ", & $25 \cdot 8$ & ", & $"$ \\
\hline
\end{tabular}

Twenty-five c.c. of the same decinormal ferric chloride, reduced and titrated by Reinhardt's method, required $25 \cdot 1$ c.c. permanganate. In order to give the method of Fresenius every chance, the ratio of acid to iron in these experiments was kept as low as we find it can be kept in practice.

As has been said in the introduction, Fresenius gave no practical example of this method, but of his second method he gave two examples which he believed to be practical. But here, again, he dissolved iron in sulphuric acid, and added the resulting ferrous solution, without any simultaneous addition of hydrochloric acid, to the contents of the vessel in which the preliminary experiment had been made.

\section{Conclusions.}

The method of Fresenius gives erroneous results, and appears to have been founded on a misconception, and never until now put to a practical test.

The method of Reinhardt, though empirical and dependent for its successful application on somewhat rigid uniformity of procedure, is yet capable of yielding results little, if at all, inferior in accuracy to those given by the best volumetric methods.

Our work was undertaken with a practical object, and, though theoretical considerations helped us in the choice of experimental methods, we do not propose to add to the mass of theory which has been woven around this subject. We may, however, point out that our results are consistent with the theory of Manchot and Wilhelms (Annalen, 1902, 325, 93), and that those recorded in Table II. appear to lend additional support to their view.

We take this opportunity of expressing our thanks to Mr. R. F. Easton for much useful help in the conduct of the large number of experiments which were found necessary to the completion of this work.

\section{Discussion.}

Mr. W. C. Birch said he had had occasion to work on this subject, and the results might perhaps be interesting, as bearing on the action of the manganous sulphate, which was an essential constituent of the Zimmermann-Reinhardt reagent. In titrating with that reagent there was a distinct, though slight, evolution of chlorine, and a brown colour was produced in the solution even by a quite small excess of permanganate over the quantity required to oxidise all the iron present. These brown solutions were very similar in appearance to those described by Pickering (J. Chem. Soc., 1879, 42,654), which were produced by the action of hydrochloric acid on any of the higher oxides of manganese. Unfortunately, it was not possible to isolate this brown colouring matter from such dilute solutions as were met with in volumetric analysis; but, by blowing hydrochloric acid into a concentrated solution of potassium permanganate, and cooling the solution with 
ice and salt, it was possible to isolate a crystalline double salt having the formula $\mathrm{MnCl}_{3} 2 \mathrm{KCl}$. $\mathrm{H}_{2} \mathrm{O}$. The reaction which caused the formation of this compound could perhaps be represented by the equation

$$
\mathrm{K}_{2} \mathrm{Mn}_{2} \mathrm{O}_{8}+16 \mathrm{HCl}=2 \mathrm{KCl}+2 \mathrm{MnCl}_{3}+8 \mathrm{H}_{2} \mathrm{O}+4 \mathrm{Cl}_{2} .
$$

It had been shown by Rice ( $J$. Chem. Soc., 1898, 74, 260) that manganous chloride reacted, though but slowly, with free chlorine at the ordinary temperature to produce $\mathrm{MnCl}_{3}$. He thought it fair to assume that under the conditions of the Zimmermann-Reinhardt titration the chlorine liberated would be in a nascent state, and would therefore be more active chemically than chlorine in the free state; and if that were so, it was to be expected that the reaction between the manganous sulphate or manganous chloride and the chlorine would go on more quickly than under the conditions of Rice's experiments. The Zimmermann-Reinhardt reagent, therefore, provided every chance for the chlorine to enter into combination before escaping from the solution, and to remain in the solution in a state in which it was able to oxidise the remainder of the ferrous iron. This was shown by the following simple experiment which he had made: About 10 c.c. of concentrated hydrochloric acid, with a few c.c. of potassium permanganate, were placed in one flask, and in another flask the same quantities of these reagents, with an excess of manganous sulphate, a current of air being aspirated through both flasks, and the evolved chlorine collected in potassium iodide, and titrated with thiosulphate. The chlorine evolved from the flask not containing manganous sulphate was equivalent to 38.1 c.c. of decinormal thiosulphate solution, while that from the flask containing manganous sulphate required only 0.5 c.c. The aspiration of air was carried on for two hours in the case of the first solution and two and a half hours in the case of the second. This might enable a mental picture to be formed of the part played. by the manganous sulphate in the Zimmermann-Reinhardt reagent.

Mr. Chapman said that palladium-hydrogen was preferable to stannous chloride as a reducing agent. One of its advantages was that nothing was introduced to. interfere with the indicator. The initial expense of the palladium was certainly somewhat large, but not so large as to be of really serious moment.

The Chatrman (Mr. Richmond) remarked that it seemed clear, from the work of the authors and of Mr. Birch, that what happened when ferrous solutions were titrated with permanganate in presence of hydrochloric acid was that, while some of the permanganate was used up in oxidising the iron, a portion of it also was used in oxidising some of the oxides of manganese, to form higher oxides, which then formed unstable chlorides, and the speed of the former reaction was greater than that of the latter. That being so, the precaution which the authors recommended of adding the permanganate drop by drop, so as to keep the concentration of the permanganate in all parts of the solution very low indeed, was obviously a wise one. He thought that the difficulty of using bichromate was not so great as had been suggested, especially if the titration was made by artificial light, a practice which he personally much preferred.

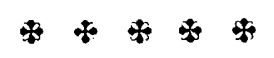

